\title{
Awareness of Bisphosphonate Use and Its Dental Complications among the Dental Practitioners
}

\author{
${ }^{1}$ Gauri Kokane, ${ }^{2}$ Sunil Sidana, ${ }^{3}$ Jyotsna Galinde, ${ }^{4}$ Imran Khalid
}

\begin{abstract}
Objectives: To assess the awareness, among dental practitioners, of the use of bisphosphonates (BP) and its complications related to dental extractions as well as to outline protocol for its prevention and management.
\end{abstract}

Materials and methods: A cross-sectional descriptive study was carried out in department of oral and maxillofacial surgery, MGM Dental College and Hospital, Kamothe, Navi Mumbai, in December 2013. Data collection was carried out by a selfadministered questionnaire distributed among interns, postgraduate students and staff members at the hospital.

Results: Total of 159 people were included in to the study. Out of which 95 were interns, 25 were postgraduate students and 39 were staff members. Four out of 39 staff members, six postgraduate students out of 25 and 18 out of 99 interns were unaware of trade name of drug alendronate as Fosamax. Eight staff out of 39 , six postgraduate students of 25 and 23 interns out of 95 were unaware of term BRONJ. Thirty-three out of 39 staff, 20 out of 25 postgraduate student and 81 out of 95 interns were aware of clinical use of BP. Thirty-eight out of 39 staff, 23 out of 25 postgraduate student and 93 out of 95 interns were familiar with BP. Fourteen out of 39 staff, three out of 25 postgraduate students and 25 out of 95 interns were aware of guidelines on treating patients on BP. Thirty-seven out of 39 staff, four out of 25 postgraduate students and 21 out of 95 interns have asked patients about past history of BP use.

Conclusion: There is a lack of complete awareness about $\mathrm{BP}$ use, its dental complications and prevention and treatment strategies for BP-related osteoradionecrosis of jaws. Bisphosphonates and its related dental complications and management should be included in the undergraduate dental curriculum.

Keywords: BRONJ, Bisphosphonates, Osteoradionecrosis, Dental.

How to cite this article: Kokane G, Sidana S, Galinde J, Khalid I. Awareness of Bisphosphonate Use and Its Dental Complications among the Dental Practitioners. J Contemp Dent 2015;5(1):1-6.

Source of support: Nil

Conflict of interest: None

\footnotetext{
${ }^{1}$ Postgraduate Student, ${ }^{2}$ Professor

${ }^{3}$ Professor and Head, ${ }^{4}$ Reader

${ }^{1-4}$ Department of Oral and Maxillofacial Surgery, MGM Dental College and Hospital, Navi Mumbai, Maharashtra, India
}

Corresponding Author: Gauri Kokane, Postgraduate Student Department of Oral and Maxillofacial Surgery, MGM Dental College and Hospital, Navi Mumbai, Maharashtra, India, Phone: 02227433185, e-mail: gaurikkn@gmail.com

\section{INTRODUCTION}

Bisphosphonates (BP) are primarily used in the treatment and management of cancer-related conditions, including hypercalcemia of malignancy, skeletal-related events associated with bone metastases in the context of solid tumors, such as breast cancer, prostate cancer and lung cancer, and management of lytic lesions in the setting of multiple myeloma. Also, used in prevention and treatment of bone fragility diseases, like the osteitis deformans ('Paget's disease of bone'), primary hyperparathyroidism, osteogenesis imperfecta, fibrous dysplasia, and other conditions that feature bone fragility, such as osteoporosis, osteopenia, etc. ${ }^{1}$ Bisphosphonates do not improve cancer-specific survival, but it has a significant positive effect on the quality of life for patients with advanced cancer involving the skeleton.

Bisphosphonate-related osteoradionecrosis of jaws (BRONJ) is one of the major complications of BS therapy. In 2003, Marx reported BRONJ or bisphosphonateinduced osteonecrosis of the jaw (BIONJ), as a side-effect of BP treatment. ${ }^{2} \mathrm{BRONJ}$ lesions are defined as exposed necrotic bone without evidence of healing for at least 8 weeks in the maxillofacial area in patients with a history of BP use without a history of head and neck irradiation. ${ }^{3}$ An incidence of 1 in 140,000 person life years of exposure associated with the orally administered drugs was originally based on BRONJ cases reported to pharmaceutical companies. The risk of developing BON increased more than four-fold when individuals underwent a dental extraction. It is necessary to create the awareness among dental practitioners and budding dentists regarding the $\mathrm{BRONJ}$, its prevention and treatment protocol. Purpose of this study is to assess the awareness of dentists regarding $\mathrm{BP}$ and its dental-related complications and to outline the prevention and treatment protocol. This study was carried out in MGM Dental College and Hospital, Kamothe, Navi Mumbai, in December 2013.

\section{MATERIALS AND METHODS}

Interns, Postgraduate students and staff members of MGM Dental College and Hospital were included in the study. Questionnaire was designed to assess the awareness with regards to BP use, its complications and management. A total of 159 individuals participated in 
the study, out of which 95 were interns, 25 were postgraduate students and 39 were staff members from various departments (oral medicine, radiology and diagnosis, oral pathology, periodontics, prosthodontics, orthodontics, pedodontics, oral and maxillofacial surgery).

\section{RESULTS}

A total of 159 people were included in to the study, out of which 39 were staff, 25 were postgraduate students, and 95 were interns. Four out of 39 staff members (10.25\%), six postgraduate students out of $25(24 \%)$ and 18 out of 99 (18.18\%) interns were unaware of trade name of drug alendronate as Fosamax. Eight staff out of 39 (20.51\%), six postgraduate students of $25(24 \%)$ and 23 interns of 95 (24.21) were unaware of term BRONJ. Thirty-three out of $39(84.61 \%), 20$ out of $25(80 \%)$ and 81 out of $95(85.26 \%)$ were aware of clinical use of BP. Thirty-eight out of 39 $(97.23 \%), 23$ out of $29(79.31 \%)$ and 93 out of $95(97.89 \%)$ were familiar with BP. Fourteen out of 39 (35.89\%) staff, 3 out of $25(10.34 \%)$ postgraduate students and 25 out of $95(26.31 \%)$ interns were aware of guidelines on treating patients on BP. Thirty seven out of $39(94.87 \%)$ staff, 4 out of $25(16 \%)$ postgraduate students and 21 out of 95 (22.10\%) interns have asked patients about history of BP use (Graphs 1 to 8).

\section{DISCUSSION}

Bisphosphonates are said to inhibit osteoclastic bone resorption and bone remodeling by inhibiting osteoclast differentiation and function and promoting apoptosis. ${ }^{4-9}$ Since the bone turnover rate is highest in maxilla and mandible, these are the most vulnerable sites for its occurrence. ${ }^{10,11}$ Other factor in pathophysiology is inhibition of angiogenesis. Osteonecrosis itself is a form of avascular necrosis, so inbibition of angiogenesis is thought to be responsible for occurrence of osteonecrosis.

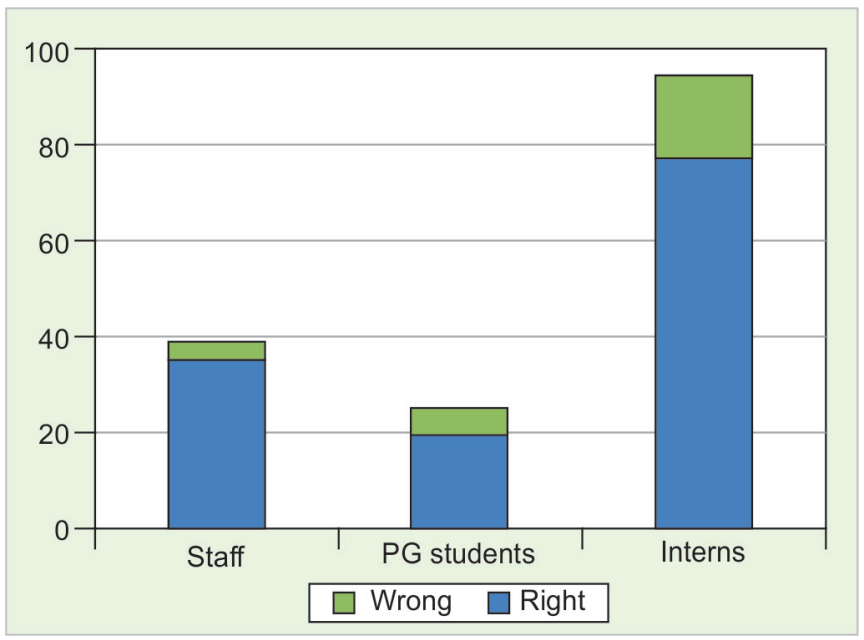

Graph 1: Fosamax (alendronate) belongs to which class of drugs
Infection and inflammation is also thought to be a cause. In the biopsy specimen, actinomyces species was found. ${ }^{12}$

In this study, 33 out of 39 (84.61\%), 20 out of 25 (68.96\%) and 81 out of $95(85.26 \%)$ were aware of clinical use of BP. Patients with metastatic disorders and hypercalcemia are treated with IV BP. ${ }^{2}$ Postmenopausal women presenting osteoporosis or osteopetrosis are prescribed with oral BP. It becomes important to take necessary precautions in these patients before undergoing any major or minor oral surgical procedure. Pamidronate, zoledronic acid are injectable $\mathrm{BP}$ and alendronate and risidronate are oral formulations. ${ }^{2}$ The typical presentation of disease is of nonhealing extraction socket or exposed jawbone with progression to sequestrum formation associated with localized swelling and purulent discharge. ${ }^{4}$ Diagnostic criterion is according to AAOMS definition, clinical and radiological findings, also biopsy, can be taken to rule out malignancy. ${ }^{2}$

It is important to understand that patients at risk of, or with established, BRONJ can also present with other common clinical conditions not to be confused with BRONJ. In this study, eight staff out of 39, six postgraduate students of 25 and 23 interns of 95 were unaware of term BRONJ and its presentation. Commonly misdiagnosed conditions can include, but are not limited to, alveolar osteitis, sinusitis, gingivitis/periodontitis, caries, periapical pathologic findings, and temporomandibular joint disorders.

\section{CLINICAL PRESENTATION}

The site of involvement may be an area of prior trauma, such as an extraction or irritation from a dental prosthesis, although spontaneous osteonecrosis also can occur. The extent of symptoms and clinical disease shows varied presentation despite of similar disease processes,

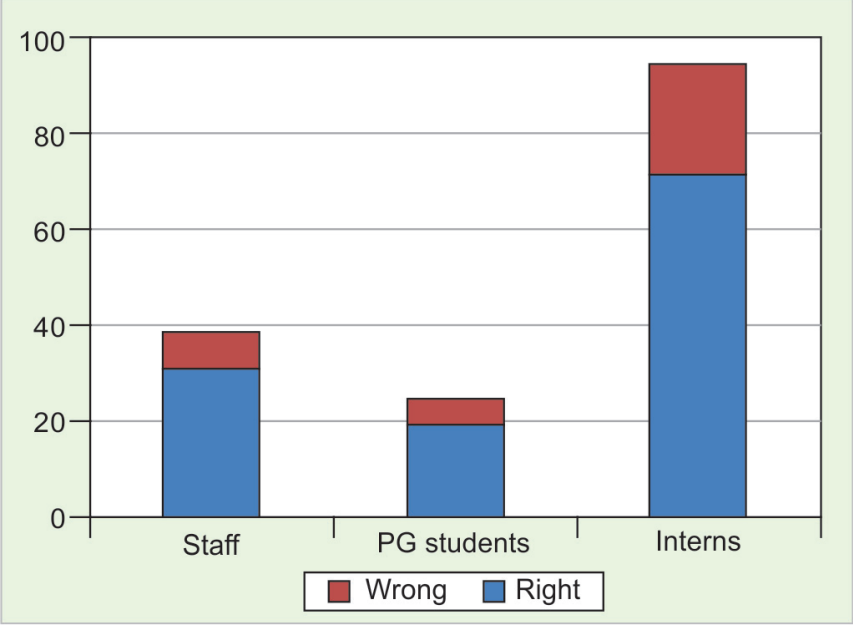

Graph 2: What is BRONJ 
bisphosphonate dose and treatment duration. A staging system was based on these observations, developed that facilitates the clinical stratification of this disease process and guides treatment strategies.

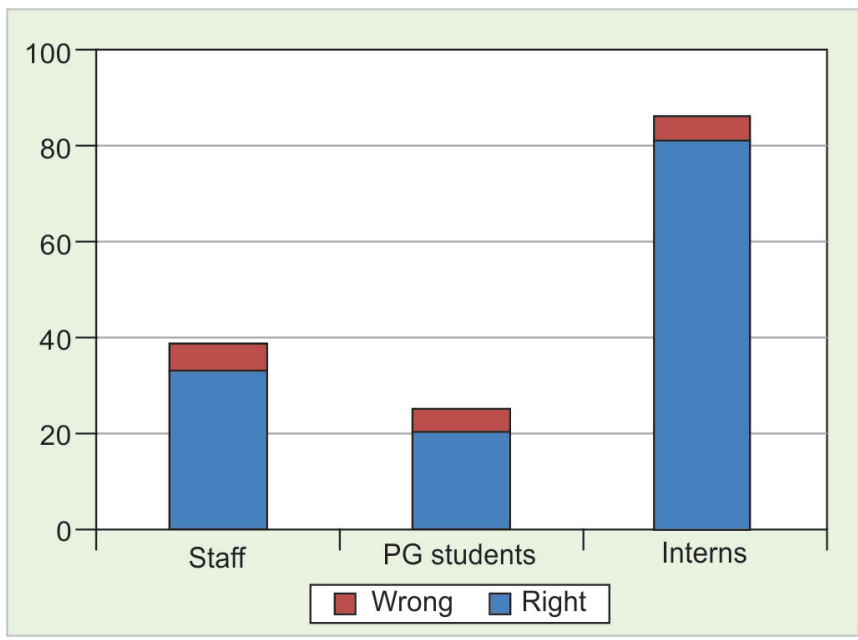

Graph 3: Bisphosphonate is used to treat

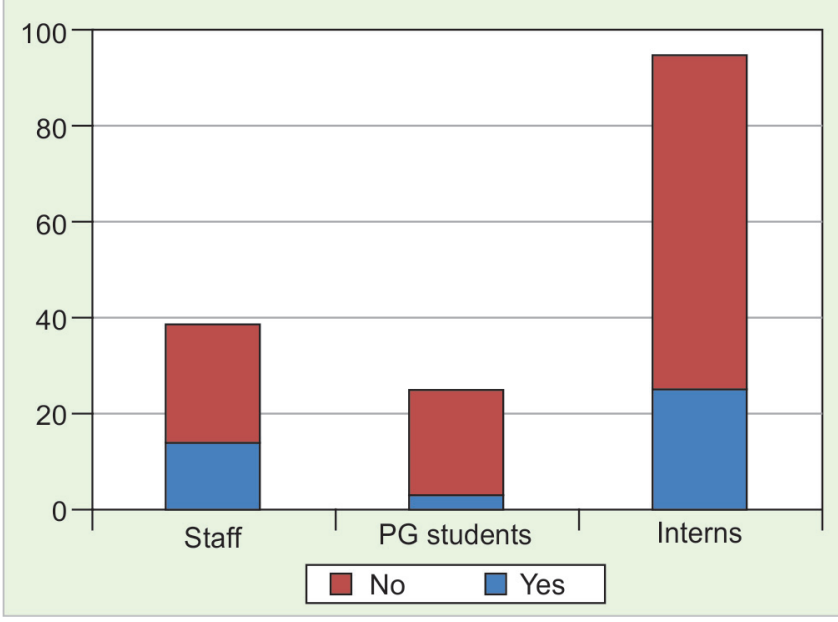

Graph 5: Are you aware of any guideline for treating patients on bisphosphonates

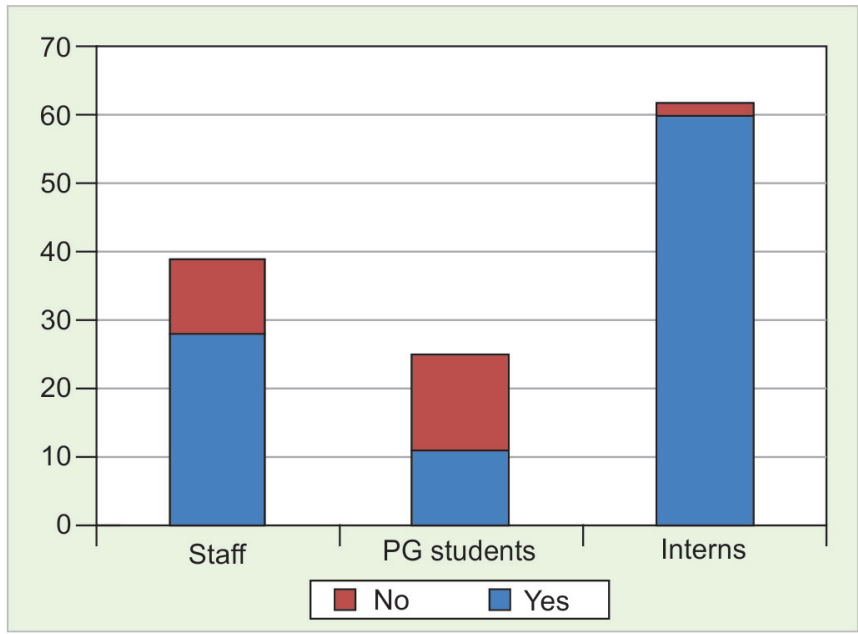

Graph 7: What is the side-effect of bisphosphonate in patients undergoing extraction
- Stage 0: Signs and symptoms short of exposed necrotic bone that might indicate a histologic necrosis or a pre necrotic state.

- Stage 1: Exposed necrotic bone that is asymptomatic.

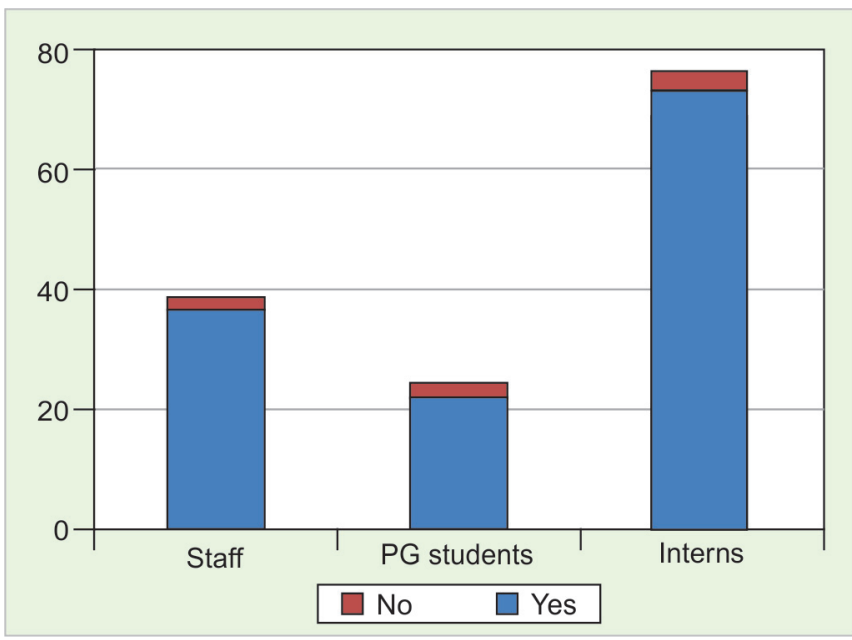

Graph 4: Have you heard about bisphosphonates

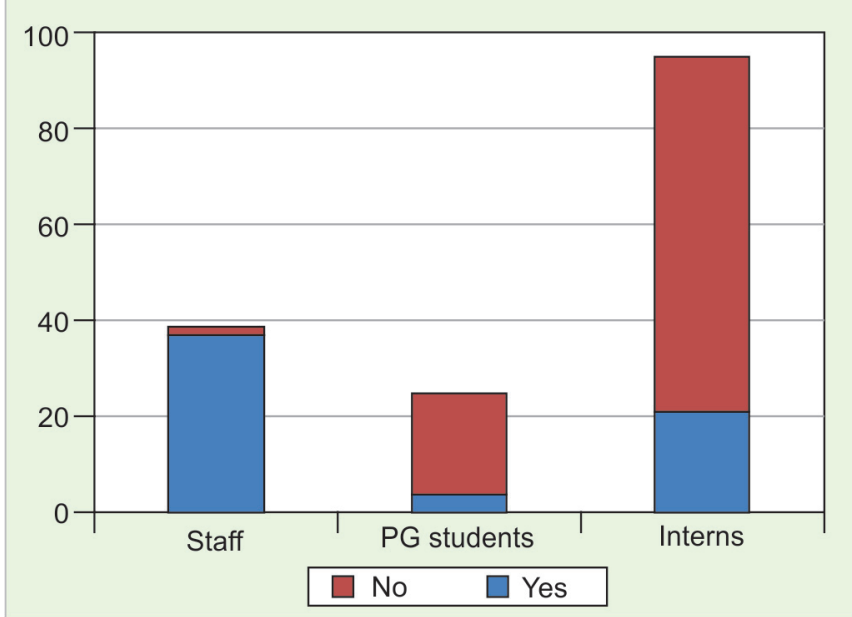

Graph 6: Did you in the past ever ask your patients for history of bisphosphonates before dental extractions

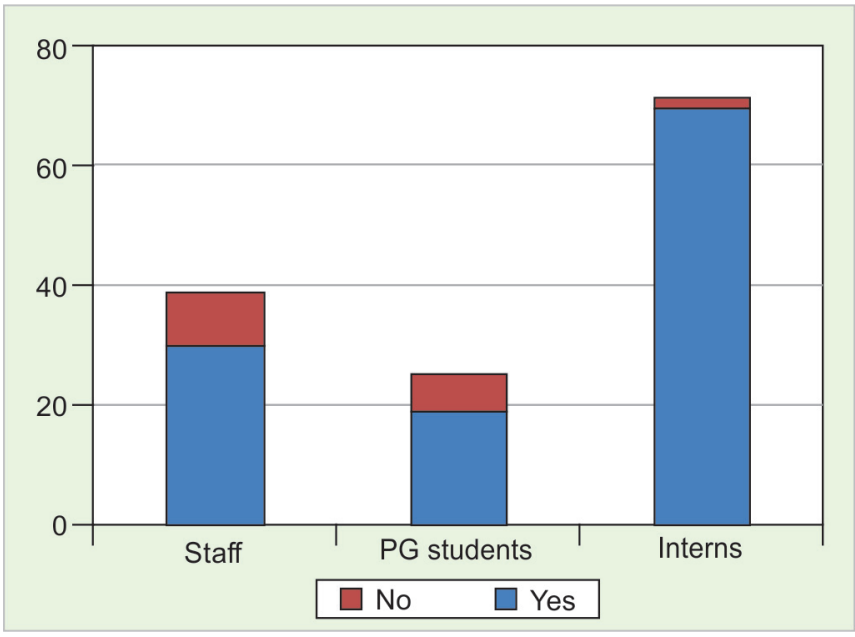

Graph 8: Can you prevent BRONJ in dental patients undergoing extractions 
- Stage 2: Exposed, necrotic bone associated with pain and adjacent or regional soft-tissue involvement.

- Stage 3: Necrotic bone with associated pain and adjacent or regional soft-tissue infection and a pathologic fracture, extraoral fistula or osteolysis extending to the inferior border.

\section{MANAGEMENT PROTOCOL}

- For prevention of BRONJ: Every patient who is going to start on BP therapy should be scanned for dental foci of infection and the focus if present should be attended and solved before the therapy starts, this will prevent the occurrence of BRONJ. ${ }^{37}$ Out of 39 staff (94.86\%), four out of 25 (16\%) postgraduate students and 21 out of 95 (22.10\%) interns have asked patients about history of BP use. Patients should be looked for caries, periodontal disease; any minor oral surgical treatment should be performed well before the BP therapy initiation. Nonrestorable teeth and teeth with poor prognosis should be extracted before hand patients with complete or partial dentures should be looked for areas of irritation over mucosa and mainly in lingual flange region. Early dental screening and initiation of appropriate treatment has found to reduce the incidence BRONJ. ${ }^{13-29}$ It is important to find out potential sites of infection along with the focus of acute infection in order to prevent further sequel which could get exacerbated when therapy is initiated. It is important to educate the patients regarding oral hygiene and regular dental checkup. Patients are asked to report immediately if any pain, swelling or exposed bone. Dimopoulos found a statistically significant, almost threefold reduction in the incidence of osteonecrosis in patients when preventive measures were applied. ${ }^{30}$

Drug holiday is another concept to prevent the risk of BRONJ. It involves temporary cessation of therapy before the invasive dental procedure, if systemic condition permits. American Association of Oral and Maxillofacial Surgeons (AAOMS) (2009) recommends discontinuing of BP therapy 3 months prior and 3 months after the invasive dental procedure. ${ }^{31}$ Patients having BP exposure for more than 4 years are at higher-risk for BRONJ, therefore, drug holiday is beneficial in such cases. It is advised to continue BP therapy in patients with BP exposure of less than 2 years. ${ }^{23}$

They note that since $50 \%$ of serum BP undergoes renal excretion the major reservoir of $\mathrm{BP}$ is the osteoclast whose life span is 2 weeks. Thus, the majority of free $\mathrm{BP}$ within the serum would be extremely low 2 months following the last dose of an oral BP and a 2-month drug-free period should be adequate prior to an invasive dental procedure. Patients physician should be contacted prior to any dental procedure with regards to discontinuation of BP drugs.

\section{TREATMENT OF BRONJ}

\section{Patients with BRONJ}

The treatment objectives for patients with an established diagnosis of BRONJ are to eliminate pain, control infection of the soft and hard tissue, and minimize the progression or occurrence of bone necrosis. ${ }^{32-37}$

- Stage 0: Provides symptomatic treatment, and conservatively manage other local factors, such as caries and periodontal disease. Systemic management may include the use of medication for chronic pain and control of infection with antibiotics, when indicated.

- Stage 1: These patients benefit from the use of oral antimicrobial rinses, such as chlorhexidine $0.12 \%$. No surgical treatment is indicated.

- Stage 2: These patients benefit from the use of oral antimicrobial rinses in combination with antibiotic therapy. It is hypothesized that the pathogenesis of BRONJ may be related to factors adversely influencing bone remodeling. Additionally, BRONJ is not due to a primary infectious etiology. Most of the isolated microbes have been sensitive to the penicillin group of antibiotics. Quinolones, metronidazole, clindamycin, doxycycline and erythromycin have been used with success in those patients who are allergic to penicillin. Microbial cultures should also be analyzed for the presence of actinomyces species of bacteria. If this microbe is isolated, the antibiotic regimen should be adjusted accordingly. In some refractory cases, patients may require combination antibiotic therapy, long-term antibiotic maintenance, or a course of intravenous antibiotic therapy.

- Stage 3: These patients benefit from debridement, including resection, in combination with antibiotic therapy, which may offer long-term palliation with resolution of acute infection and pain.

Regardless of the disease stage, mobile segments of bony sequestrum should be removed without exposing uninvolved bone. The extraction of symptomatic teeth within exposed, necrotic bone should be considered, since it is unlikely that the extraction will exacerbate the established necrotic process. General dental practitioners should seek the opinion and advice of the oncologist managing the patient with regards to discontinuation of intravenous BP therapy. Oral and maxillofacial surgeon should be involved with regards to management of BP-related osteoradionecrosis of the jaws. 


\section{CONCLUSION}

There is lack of complete awareness regarding the side effects and guidelines for management of BP-related osteoradionecrosis of the jaws. It is a very important to find out patients who are at risk of BRONJ or who have developed the same. Meticulous examination and treatment is the need. So, it is very important to develop awareness regarding BP use and its complications among the dental professionals.

\section{REFERENCES}

1. National osteoporosis society. Drug treatment UK National Osteoporosis Society. Retrieved 7 Aug 2012.

2. Hellstein JW, Marek CL. Bisphosphonate osteochemonecrosis (Bis-Phossy Jaw): is this phossy jaw of the 21st century? J Oral Maxillofac Surg 2005 May;63(5):682-689.

3. Yamazaki T, Yamori M, Ishizaki T, Asai K, Goto K, Takahashi K, Nakayama T, Bessho K. Increased incidence of osteonecrosis of the jaw after tooth extraction in patients treated with bisphosphonates: a cohort study. Int J Oral Maxillofac Surg 2012 Nov;41(11):1397-1403.

4. Altundal H, Güvener O. The effect of alendronate on resorption of the alveolar bone following tooth extraction. Int J Oral Maxillofac Surg 2004;33:286-293.

5. Baron R, Ferrari S, Russell RG. Denosumab and bisphosphonates: different mechanisms of action and effects. Bone 2011 Apr 1;48(4):677-692.

6. Lacey DL, Boyle WJ, Simonet WS, et al. Bench to bedside: elucidation of the OPG-rank-rankl pathway and the development of denosumab. Nat Rev Drug Discov 2012 May; 11(5):401-419.

7. Russell RG, Watts NB, Ebetino FH, et al. Mechanisms of action of bisphosphonates: similarities and differences and their potential influence on clinical efficacy. Osteoporos Int 2008 Jun;19(6):733-759.

8. Roelofs AJ, Thompson K, Gordon S, et al. Molecular mechanisms of action of bisphosphonates: current status. Clin Cancer Res 2006 Oct 15;12(20 Pt 2):62225-62305.

9. Russell RG, Rogers MJ. Bisphosphonates: from the laboratory to the clinic and back again. Bone 1999 Jul;25(1):97-106.

10. Marx RE, Sawatari Y, Fortin M, Broumand V. Bisphosphonateinduced exposed bone (osteonecrosis/osteopetrosis) of the jaws: risk factors, recognition, prevention and treatment. J Oral Maxillofac Surg 2005 Nov;63(11):1567-1575.

11. Marx RE, Cillo JE Jr, Ulloa JJ. Oral bisphosphonate-induced osteonecrosis: risk factors, prediction of risk using serum CTX testing, prevention and treatment. J Oral Maxillofac Surg 2007 Dec;65(12):2397-2410.

12. Hansen T, Kunkel M, Weber A, et al. Osteonecrosis of the jaws in patients treated with bisphosphonates-histomorphologic analysis in comparison with infected osteoradionecrosis. J Oral Pathol Med 2006 Mar;35(3):155-160.

13. Sonis ST, Watkins BA, Lyng GD, et al. Bony changes in the jaws of rats treated with zoledronic acid and dexamethasone before dental extractions mimic bisphosphonate-related osteonecrosis in cancer patients. Oral Oncol 2009 Feb;45(2): 164-172.

14. Nicolatou-Galitis O, Migkou M, Psyrri A, et al. Gingival bleeding and jaw bone necrosis in patients with metastatic renal cell carcinoma receiving sunitinib: report of two cases with clinical implications. Oral Surg Oral Med Oral Pathol Oral Radiol 2012 Feb;113(2):234-238.

15. Smidt-Hansen T, Folkmar TB, Fode K, et al. Combination of zoledronic acid and targeted therapy is active but may induce osteonecrosis of the jaw in patients with metastatic renal cell carcinoma. J Oral Maxillofac Surg 2013 Sep;71(9):1532-1540.

16. Fehm T, Beck V, Banys M, et al. Bisphosphonate-induced osteonecrosis of the jaw (ONJ). Incidence and risk factors in patients with breast cancer and gynecological malignancies. Gynecol Oncol 2009 Mar;112(3):605-609.

17. Kyrgidis A, Vahtsevanos K, Koloutsos G, etal. Bisphosphonaterelated osteonecrosis of the jaws: a case-control study of risk factors in breast cancer patients. J Clin Oncol 2008 Oct 1;26(28):4634-4638.

18. Sivolella S, Lumachi F, Stellini E, et al. Denosumab and antiangiogenetic drug-related osteonecrosis of the jaw: an uncommon but potentially severe disease. Anticancer Res 2013 May;33(5):1793-1797.

19. Epstein MS, Epstein JB, Ephros HD. The effects of osteoclast modifiers on the oral cavity: a review for prescribers. Curr Opin Support Palliat Care 2012 Sep;6(3):337-341.

20. Vescovi P, Merigo E, Meleti M, et al. Bisphosphonates-related osteonecrosis of the jaws: a concise review of the literature and a report of a single-centre experience with 151 patients. J Oral Pathol Med 2012 Mar;41(3):214-221.

21. Schubert M, Klatte I, Linek W, et al. The saxon bisphosphonate register-therapy and prevention of bisphosphonaterelated osteonecrosis of the jaws. Oral Oncol 2012 Apr;48(4): 349-354.

22. Shannon J, Modelevsky S, et al. Bisphosphonates and osteonecrosis of the jaw. J Am Geriatr Soc 2011 Dec;59(12):2350-2355.

23. Lo JC, O'Ryan F, Yang J, et al. Oral health considerations in older women receiving oral bisphosphonate therapy. J Am Geriatr Soc 2011 May;59(5):916-922.

24. Hellstein JW, Adler RA, Edwards B, et al. Managing the care of patients receiving antiresorptive therapy for prevention and treatment of osteoporosis: executive summary of recommendations from the American Dental Association Council on Scientific Affairs. J Am Dent Assoc 2011 Nov;142(11): 1243-1251.

25. Patel V, McLeod NM, Rogers SN, et al. Bisphosphonate osteonecrosis of the jaw-a literature review of UK policies versus international policies on bisphosphonates, risk factors and prevention. Br J Oral Maxillofac Surg 2011 Jun;49(4):251-257.

26. Atalay B, Yalcin S, Emes Y, et al. Bisphosphonate-related osteonecrosis: laser-assisted surgical treatment or conventional surgery? Lasers Med Sci 2011 Nov;26(6):815-823.

27. Aapro M, Saad F, Costa L. Optimizing clinical benefits of bisphosphonates in cancer patients with bone metastases. Oncologist 2010;15(11):1147-1158.

28. Fehm T, Felsenberg D, Krimmel M, et al. Bisphosphonateassociated osteonecrosis of the jaw in breast cancer patients: recommendations for prevention and treatment. Breast 2009 Aug;18(4):213-217.

29. Walter C, Al-Nawas B, du Bois A, et al. Incidence of bisphosphonate-associated osteonecrosis of the jaws in breast cancer patients. Cancer 2009 Apr 15;115(8):1631-1637.

30. Khan AA, Sandor GK, Dore E, et al. Bisphosphonate associated osteonecrosis of the jaw. J Rheumatol 2009 Mar;36(3): 478-490. 
31. Dickinson M, Prince HM, Kirsa S, et al. Osteonecrosis of the jaw complicating bisphosphonate treatment for bone disease in multiple myeloma: an overview with recommendations for prevention and treatment. Int Med J 2009 May;39(5): 304-316.

32. Edwards BJ, Hellstein JW, Jacobsen PL, et al. Updated recommendations for managing the care of patients receiving oral bisphosphonate therapy: an advisory statement from the American Dental Association Council on Scientific Affairs. J Am Dent Assoc 2008 Dec;139(12):1674-1677.

33. Abu-Id MH, Warnke PH, Gottschalk J, et al. 'Bis-phossy jaws'-high and low risk factors for bisphosphonate-induced osteonecrosis of the jaw. J Craniomaxillofac Surg 2008 Mar; 36(2):95-103.
34. Kyle RA, Yee GC, Somerfield MR, et al. American Society of clinical oncology 2007 clinical practice guideline update on the role of bisphosphonates in multiple myeloma. J Clin Oncol 2007 Jun 10;25(17):2464-2472.

35. Dimopoulos MA, Kastritis E, Bamia C, et al. Reduction of osteonecrosis of the jaw (ONJ) after implementation of preventive measures in patients with multiple myeloma treated with zoledronic acid. Ann Oncol 2009 Jan;20(1):117-120.

36. Khan AA, Morrison A, Hanley DA, et al. International consensus on diagnosis and management of osteonecrosis of the Jaw. J Bone Miner Res 2015 Jan;30(1):3-23.

37. Damm DD, Jones DM. Bisphosphonate-related osteonecrosis of the jaws: a potential alternative to drug holidays. Gen Dent 2013 Aug;61(5):33-38 